Correspondence

\title{
An unusual trigger causing Takotsubo Syndrome牥
}

\author{
Glória Abreu*, Sérgia Rocha, Nuno Bettencourt, Pedro Azevedo, Catarina Vieira, Catarina Rodrigues, \\ Carina Arantes, Carlos Braga, Juliana Martins, Jorge Marques
}

Cardiology Department, Hospital de Braga, Braga, Portugal

\section{A R T I C L E I N F O}

\section{Article history:}

Received 18 July 2016

Accepted 7 August 2016

Available online 09 August 2016

Takotsubo Syndrome is an important differential diagnosis of acute coronary syndrome and this entity still represents a process of exclusion. Our case reports the possibility of an association between Takotsubo Syndrome and acute myocardial infarction involving complete different settings.

A 68-year-old woman with previous history of hypertension, dyslipidemia, hypothyroidism and ischemic colitis diagnosed 5 years before and treated with surgery was admitted in emergency department due to a crushing chest pain irradiating to the back, with acute onset at rest. It was associated with nausea, diaphoresis and dyspnoea and lasted more than $1 \mathrm{~h}$. She denied other symptoms, any previous stressful event or infectious context. On admission, physical examination was normal. The electrocardiogram (ECG) revealed sinusal rhythm, ST segment elevation in inferior and anterior leads (Fig. 1 panel A), reason why an emergent coronary angiography was performed. Besides coronary irregularities, it depicted first obtuse marginal bifurcated and occluded distally in both branches (thin vessels). Ventriculography disclosed akinesis/dyskinesis of mid to apical segments of left ventricle (apical ballooning), sparing basal segments (Fig. 1 panels B-D; Videos 1 and 2). The transthoracic echocardiography (TTE), performed immediately after, calculated left ventricle ejection fraction (LVEF) in 33\%, supporting the same wall motion abnormalities previously described, without significant valvular diseases. Blood analysis showed a peak of troponin I level of $61.30 \mathrm{ng} / \mathrm{mL}$, NT-proBNP $34347 \mathrm{pg} / \mathrm{mL}$. Evolution of ECG abnormalities was marked by T wave inversion in I, aVL, II, III, aVF and V2-V6 leads. Seven days after, a cardiac magnetic resonance (CMR) imaging was performed, which revealed mild left ventricular dysfunction, hypokinesia of mid to apical segments of all walls, except in mid to apical inferolateral segments, which were akinetic. T2-weighted CMR showed intramyocardial edema in mid to apical segments of all walls,

\footnotetext{
is The work was performed in Cardiology Department of Braga Hospital, Braga, Portugal * Corresponding author at: Cardiology Department, Hospital de Braga, Sete Fontes - São Victor, 4710-243 Braga, Portugal.

E-mail address: gloriappabreu@gmail.com (G. Abreu).
}

consistent to the distribution of motion abnormalities. CMR disclosed subendocardial late gadolinium enhancement in the mid and distal inferolateral segments, as well as an area of low signal intensity suggestive of microvascular obstruction. Data compatible with acute phase of coronary syndrome in obtuse marginal territory and Takotsubo Syndrome (Fig. 1 panels E-I, Video 3).

There were no in hospital complications. She was discharged asymptomatic, treated with ACE-inhibitor, beta-blocker, statin and anticoagulant therapy with the diagnosis Takotsubo Syndrome secondary to embolic myocardial infarction. Five months later, she repeated CMR that depicted preserved left ventricular function. The only motion abnormalities observed were akinesis in mid inferolateral segment, where it was observed transmural late gadolinium enhancement, and hypokinesia in mid segment of inferior wall and distal segment of lateral wall, those segments showing subendocardial late gadolinium enhancement. Intramyocardial edema was not evident in this exam (Fig. 2 panels A-E, Video 4).

Takotsubo Syndrome is an acute and frequently reversible heart failure syndrome, whose underlying pathophysiological mechanisms are not well understood. From vast mechanisms proposed, the most accepted are catecholaminergic storm, microvascular dysfunction, multivessel spasm and aborted myocardial infarction with spontaneous recanalization [1].

The new Takotsubo Syndrome diagnostic criteria proposed in 2015 by Heart Failure Association of the European Society of Cardiology are (1) transient regional wall motion abnormalities frequently, but not always, preceded by a stressful trigger; (2) the regional wall motion abnormalities usually extend beyond a single epicardial vascular distribution, (3) the absence of culprit atherosclerotic coronary artery disease including acute plaque rupture, thrombus formation, and coronary dissection or other pathological conditions to explain the pattern of temporary LV dysfunction observed (e.g. hypertrophic cardiomyopathy, viral myocarditis); (4) new and reversible ECG abnormalities; (5) significantly elevated serum natriuretic peptide (BNP or NT-proBNP) during the acute phase; (6) positive but relatively small elevation in cardiac troponin measured with a conventional assay and (7) recovery of ventricular systolic function on cardiac imaging at follow-up (3-6 months). It highlights the possibility of secondary Takotsubo Syndrome after acute medical and surgical conditions, but the acute coronary syndrome does not appear listed [1].

In this case, the patient experienced an embolic inferolateral acute myocardial infarction. To support our diagnosis, we had the history of cardiovascular risk factors, the electrocardiographic evolution, the 

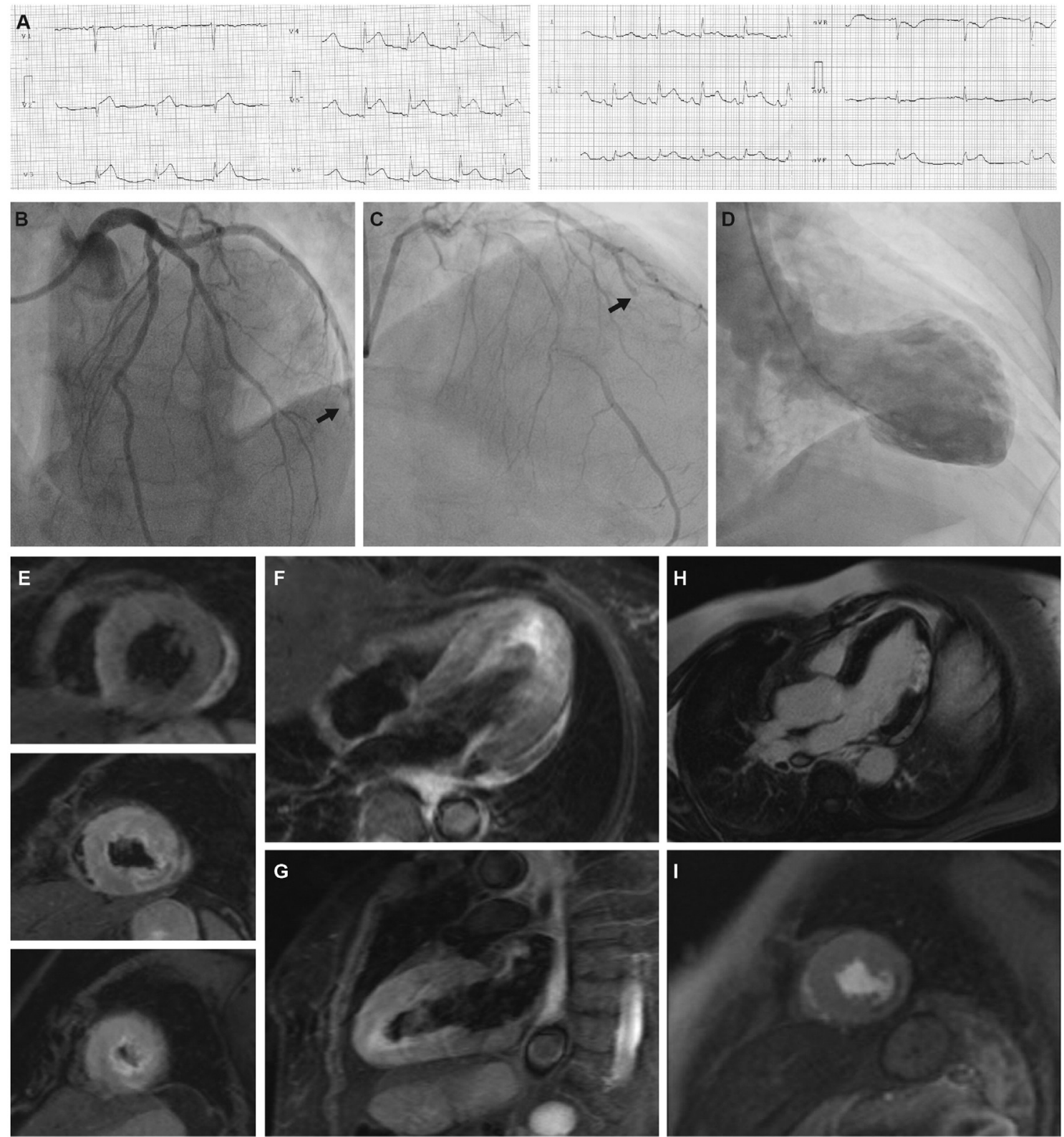

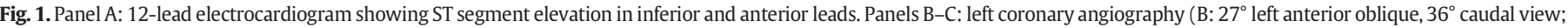

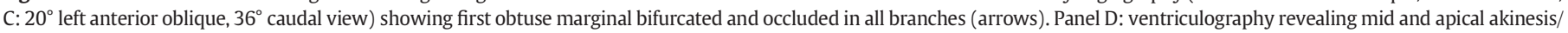

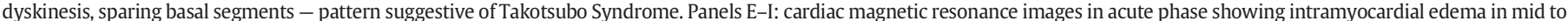

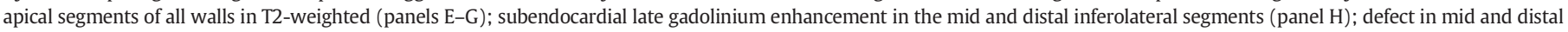
inferolateral segments in perfusion at rest (Panel I).

coronarography showing occlusion of two branches of first obtuse marginal, the level of troponin reached and the presence of subendocardial scar in the mid and apical inferolateral segments and an area of low signal intensity suggestive of microvascular obstruction on CMR.

At the same time, our patient indeed depicted findings consistent with typical mid-apical Takotsubo Syndrome which were ventriculography and transthoracic echocardiography showing the classic pattern of midapical akinesis extending beyond a single epicardial coronary distribution on baseline. CMR performed 7 days later depicted typical mid-apical ballooning, global systolic function recovery, and intramyocardial edema in mid to apical segments. No stress factor was identified apart from myocardial ischemia, so it would be reasonable to consider this case as midapical Takotsubo Syndrome triggered by an embolic inferolateral myocardial infarction.

Our theory is in line with increasing evidence in literature supporting the likelihood of a Takotsubo component in the situation of acute coronary syndrome [2-7]. In our revision of literature, we found only three cases of Takotsubo Syndrome after a well-documented myocardial infarction [5-7]. It is supported the idea of an acute coronary syndrome as one of the various stress conditions [4]. The definition of Takotsubo Syndrome by the new Takotsubo Syndrome diagnostic criteria [1], where it is stated the absence of culprit atherosclerotic coronary artery disease including acute plaque rupture, thrombus formation and coronary dissection, can be questioned as, regarding this case, it can occur secondarily a well-documented coronary obstruction (thrombus formation).

Supplementary data to this article can be found online at http://dx. doi.org/10.1016/j.ijcard.2016.08.162.

\section{Conflict of interest}

The authors report no relationships that could be construed as a conflict of interest. 

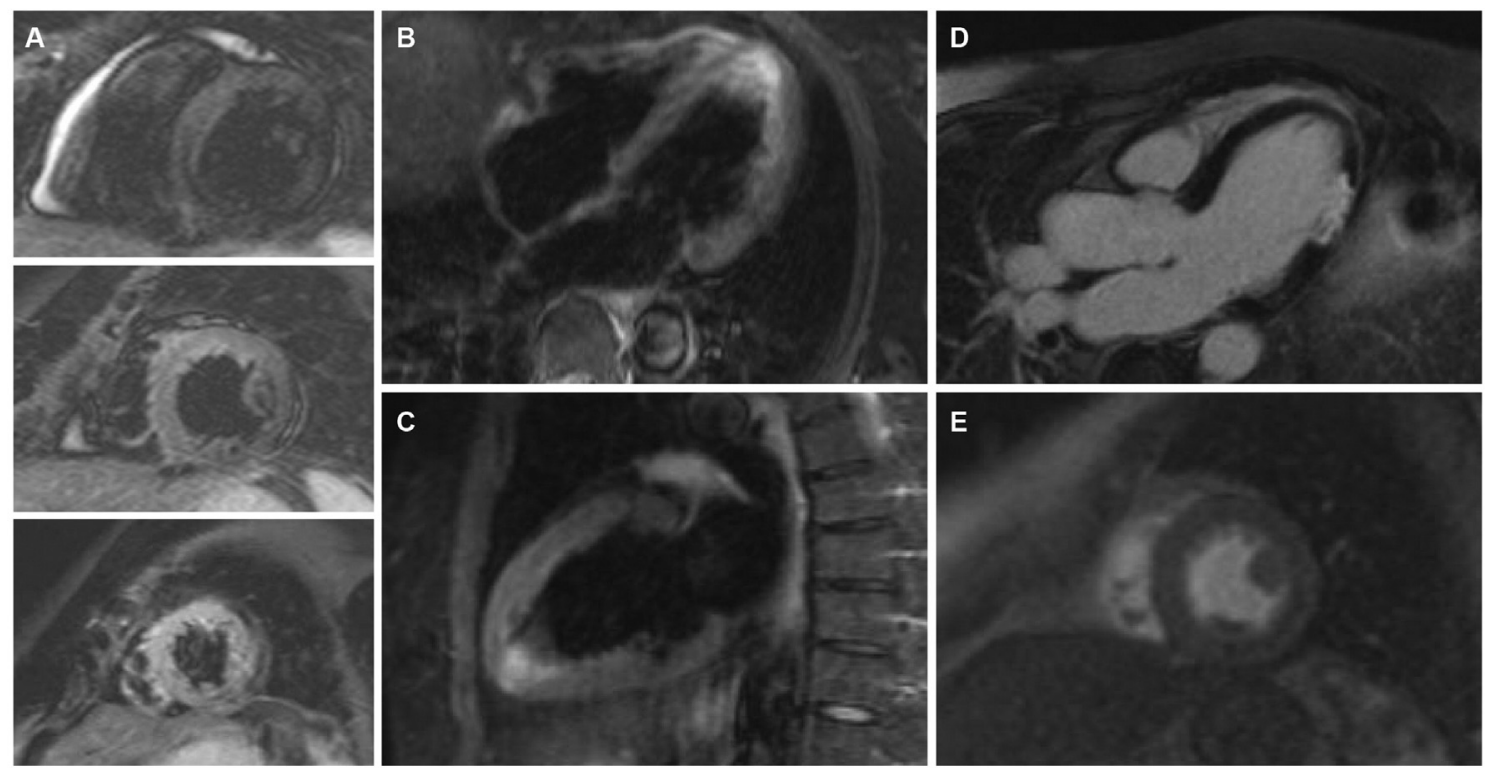

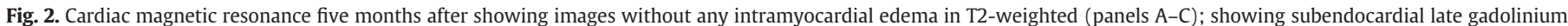
enhancement in the mid and distal inferolateral segments (panel D) and defect in mid and distal inferolateral segments, in perfusion at rest (panel E).

\section{References}

[1] A.R. Lyon, E. Bossone, B. Schneider, A.R. Lyon, E. Bossone, B. Schneider, et al., Current state of knowledge on Takotsubo syndrome: a Position Statement from the Taskforce on Takotsubo Syndrome of the Heart Failure Association of the European Society of Cardiology, Eur. J. Heart Fail. 18 (1) (Jan 2016) 8-27, http://dx.doi.org/10.1002/ ejhf.424 Epub 2015 Nov 9.

[2] L.C. Napp, J.R. Ghadri, J. Bauersachs, C. Templin, Acute coronary syndrome or Takotsubo cardiomyopathy: the suspect may not always be the culprit, Int. J. Cardiol. 187 (2015) 116-119.

[3] T. Chao, J. Lindsay, S. Collins, L. Woldeyes, S.B. Joshi, D.H. Steinberg, et al., Can acute occlusion of the left anterior descending coronary artery produce a typical "takotsubo" left ventricular contraction pattern? Am. J. Cardiol. 104 (2009) 202-204.
[4] J.E. Madias, Coronary artery disease/Takotsubo syndrome vs. acute coronary syndromes/Takotsubo syndrome, and their physical/emotional triggers, Int. J. Cardiol. 189 (2015) 279-280.

[5] N. Messas, T. Caspar, L. Jesel, S. Hess, M. Girardey, B. Radulescu, et al., Takotsubo cardiomyopathy triggered by ischemic injury: when lateral myocardial infarction precipitate apical ballooning syndrome, Int. J. Cardiol. 202 (Jan 1 2016) 858-860, http://dx.doi.org/10.1016/j.ijcard.2015.10.065 Epub 2015 Oct 8.

[6] M.N. Menezes, D. Silva, A.G. Almeida, F.J. Pinto, D. Brito, A rare case of concomitant stress (takotsubo) cardiomyopathy and acute myocardial infarction, Rev. Port. Cardiol. 34 (7-8) (Jul-Aug 2015) 499.e1-3, http://dx.doi.org/10.1016/j.repc.2015. 01.007 Epub 2015 Jul 9.

[7] B. Redfors, T. Råmunddal, Y. Shao, E. Omerovic, Takotsubo triggered by acute myocardial infarction: a common but overlooked syndrome? J. Geriatr. Cardiol. 11 (2) (Jun 2014) 171-173, http://dx.doi.org/10.3969/j.issn.1671-5411.2014.02.001. 